\title{
Integrative genomic analyses on GLI1: Positive regulation of GLI1 by Hedgehog-GLI, TGFß-Smads, and RTK-PI3K-AKT signals, and negative regulation of GLI1 by Notch-CSL-HES/HEY, and GPCR-Gs-PKA signals
}

\author{
YURIKO KATOH ${ }^{1}$ and MASARU KATOH ${ }^{2}$ \\ ${ }^{1}$ M\&M Medical BioInformatics, Hongo 113-0033; ${ }^{2}$ Genetics and Cell Biology Section, \\ National Cancer Center, Tokyo 104-0045, Japan
}

Received April 22, 2009; Accepted May 19, 2009

DOI: 10.3892/ijo_00000328

\begin{abstract}
GLI family members are zinc-finger transcription factors, which are involved in embryogenesis and carcinogenesis through transcription regulation of GLII, CCNDI, CCND2, FOXA2, FOXC2, RUNX2, SFRP1, and JAG2. GLII transcription is upregulated in a variety of human tumors, such as basal cell carcinoma, lung cancer, breast cancer, gastric cancer, pancreatic cancer, and esophageal cancer. Hedgehog signaling via Smoothened cascade and receptor tyrosine kinase (RTK) signaling via PI3K-AKT cascade induce stabilization of GLI1 protein, whereas G-protein coupled receptor (GPCR) signaling via Gs-PKA cascade induces degradation of GLI1 protein. Here we report integrative genomic analyses of the GLII gene. The GLII and ARHGAP9 genes are located in a tail-to-tail manner with overlapping 3 '-ends. ARHGAP9 was expressed in bone marrow, spleen, thymus, monocytes, and macrophages, whereas GLII was almost undetectable in normal tissues or cells with predominant ARHGAP expression. Because overlapping sense and anti-sense transcripts are annealed to each other to give rise to doublestranded RNAs functioning as endogenous RNAi, GLII expression might be negatively regulated by ARHGAP 9 transcripts. GLI-binding element with one base substitution at the +1589 -bp position from the transcriptional start site (TSS) of the human GLII gene was completely conserved in chimpanzee GLII, mouse Glil, and rat Glil genes. Ten Smad-binding elements, double E-boxes for EMT regulators, and double N-boxes for HES/HEY family members within intron 1 of the human GLII gene were also conserved in mammalian GLII orthologs. GLII transcription is upregulated
\end{abstract}

Correspondence to: Dr Masaru Katoh, Genetics and Cell Biology Section, National Cancer Center Research Institute, 5-1-1 Tsukiji, Chuo Ward, Tokyo 104-0045, Japan

E-mail:mkatoh-kkr@umin.ac.jp

Key words: Hedgehog, TGFß, breast cancer, gastric cancer, pancreatic cancer, peritoneal dissemination, pleural dissemination, systems biology, bioinformatics, personalized medicine due to Hedgehog, and TGFß signaling activation, whereas GLII transcription is downregulated due to Snail/Slug, and Notch signaling activation. Together these facts indicate that Hedgehog, TGFß, and RTK signals positively regulate GLI1, and that Notch, and GsPCR signals negatively regulate the GLI1.

\section{Introduction}

Sonic Hedgehog (SHH), Indian Hedgehog (IHH), and Desert Hedgehog (DHH) are secreted-type glycoproteins with lipid modifications involved in embryogenesis, adult tissue homeostasis, and carcinogenesis (1-4). Mature Hedgehog proteins bind to Patched family receptors to activate Smoothened signaling cascade, which results in GLI-mediated transcriptional upregulation of Hedgehog target genes (5-8).

GLI1, GLI2, and GLI3 are GLI family members (9-14). GLI1 consists of zinc finger domains, and C-terminal activator domain, whereas GLI2 and GLI3 consist of N-terminal repressor domain, zinc finger domains, and C-terminal activator domain. In the absence of Hedgehog signals, GLI1 is transcriptionally repressed, GLI2 is phosphorylated for the FBXW11/BTRCP2-mediated degradation, and GLI3 is phosphorylated for the FBXW1/BTRCP1-mediated processing into repressor (10-12). In the presence of Hedgehog signals, stabilized GLI family members induce transcriptional activation of target genes, such as GLI1, CCND1, CCND2, FOXA2, FOXC2, RUNX2, SFRP1, and JAG2 (15-21). Hedgehoginduced GLII upregulation augments the Hedgehog signaling cascades through a positive-feedback mechanism.

GLII is upregulated in various types of human tumors, as summarized in Table I. GLII is amplified and overexpressed in glioma (9), rhabdomyosarcoma (22), and osteosarcoma (22). GLII is also amplified in B-cell lymphoma (23). Although GLII is almost undetectable in adult human tissue by using RNase A protection assay, GLII expression is detected in embryonal carcinoma (24). Because GLII is a representative target gene of the Hedgehog signaling cascades, GLII is upregulated in basal cell carcinoma (25), medulloblastoma (26), lung cancer (27), gastric cancer $(28,29)$, pancreatic cancer $(28,30)$, esophageal cancer $(28,31)$, breast cancer $(32)$, prostate 
Table I. Genetic alteration or expression of GLII in human tumors.

\begin{tabular}{llc}
\hline Human cancer & Expression and mechanism & Refs. \\
\hline Glioma & Gene amplification and overexpression & $(9)$ \\
Rhabdomyosarcoma & Gene amplification and overexpression & $(22)$ \\
Osteosarcoma & Gene amplification and overexpression & $(22)$ \\
B-cell lymphoma & Gene amplification & $(23)$ \\
Embryonal carcinoma & Expression & $(24)$ \\
Basal cell carcinoma & Upregulation by Hedgehog signaling & $(25)$ \\
Medulloblastoma & Upregulation by Hedgehog signaling & $(26)$ \\
Lung cancer & Upregulation by Hedgehog signaling & $(27)$ \\
Gastric cancer & Upregulation by Hedgehog signaling & $(28,29)$ \\
Pancreatic cancer & Upregulation by Hedgehog signaling & $(28,30)$ \\
Esophageal cancer & Upregulation by Hedgehog signaling \\
Breast cancer & Upregulation by Hedgehog signaling & $(28,31)$ \\
Prostate cancer & Upregulation by Hedgehog signaling & $(32)$ \\
Liver cancer & Upregulation by Hedgehog signaling & $(33)$ \\
Cervical cancer & Upregulation by Hedgehog signaling & $(34)$ \\
Endometrial cancer & Upregulation by Hedgehog signaling & $(35)$ \\
Melanoma & Upregulation by Hedgehog signaling & $(36)$ \\
Ovarian cancer & Upregulation by Hedgehog signaling & $(37)$ \\
Ewing sarcoma & Upregulation by EWS/FLI1 fusion protein & $(38)$ \\
\hline
\end{tabular}

cancer (33), liver cancer (34), cervical uterine cancer (35), endometrial uterine cancer (36), melanoma (37), and ovarian cancer (38). GLII is also upregulated based on the EWS-FLI fusion protein generated by chromosomal translocation in Ewing sarcoma (39).

The GLII core promoter, defined as a region surrounding the transcription start site (TSS), is involved in the transcriptional regulation of human GLII gene (40). Hedgehog signals induce transcriptional upregulation of mouse Glil by using GLI-binding site within intron 1 (41), whereas TWIST1/ TWIST induces transcriptional upregulation of human GLII by using E-boxes within intron 1 (42). Because proximal promoter region and intron 1 are involved in transcriptional regulation of $G L I 1$, we carried out refined integrative genomic analyses of GLI1 to elucidate the mechanisms of GLI1 upregulation in human tumors with the emphasis on the stemcell signaling network.

\section{Materials and methods}

Comparative genomic analyses. Human genome sequences corresponding to human GLI1 RefSeq (NM_005269.2) were searched for by using BLAST programs, as previously described $(43,44)$. GLII expressed sequence tags (ESTs) were also searched for to identify GLII splicing variants $(45,46)$. Conserved transcription factor-binding sites within GLII promoters were then searched for based on manual inspection, as previously described $(47,48)$.

Regulatory network analyses. The literature on Hedgehog, TGFß, Notch and receptor tyrosine kinase (RTK) signaling cascades in PubMed and Medline databases was critically evaluated to extract knowledge. The mechanisms of GLII transcription were then investigated based on our data of conserved transcription factor-binding sites within GLII regulatory regions and in-house knowledgebase of transcription factors regulated by the stem-cell signaling network.

\section{Results}

Comparative genomic analyses of GLI1. BLAST programs using GLI1 RefSeq (NM_005269.2) as a query sequence revealed that $G L I 1$ gene is located within human genome sequence AC022506.38. Human GLII gene, consisting of 12 exons, is about $12 \mathrm{~kb}$ in size, as previously reported by Liu et al (40). Human GLII gene is located between the INHBC-INHBE gene cluster and the ARHGAP9 gene at human chromosome 12q13.3. The GLI1 gene is the paralog of the GLI2 gene at human chromosome $2 \mathrm{q} 14.12$, whereas the INHBC and INHBE genes encoding Inhibin family members are paralogs of the $I N H B B$ gene neighboring the GLI2 gene (data not shown). The INHBC-INHBE-GLII locus at human chromosome 12q13.3 and the INHBB-GLI2 locus at human chromosome $2 \mathrm{q} 14.12$ are paralogous regions within the human genome.

The GLII and ARHGAP9 genes are located in a tail-totail manner with the overlapping 16 bases in their 3 '-ends (Fig. 1). ARHGAP9 is expressed in peripheral blood leukocytes, spleen, thymus (49), and also in thyroid gland, testis, bone marrow, monocytes, and macrophages (RefEX database). GLII is almost undetectable in normal tissues with predominant ARHGAP9 expression (RefEX database). Because overlapping sense and anti-sense transcripts are annealed to 
$12 q 13.3$

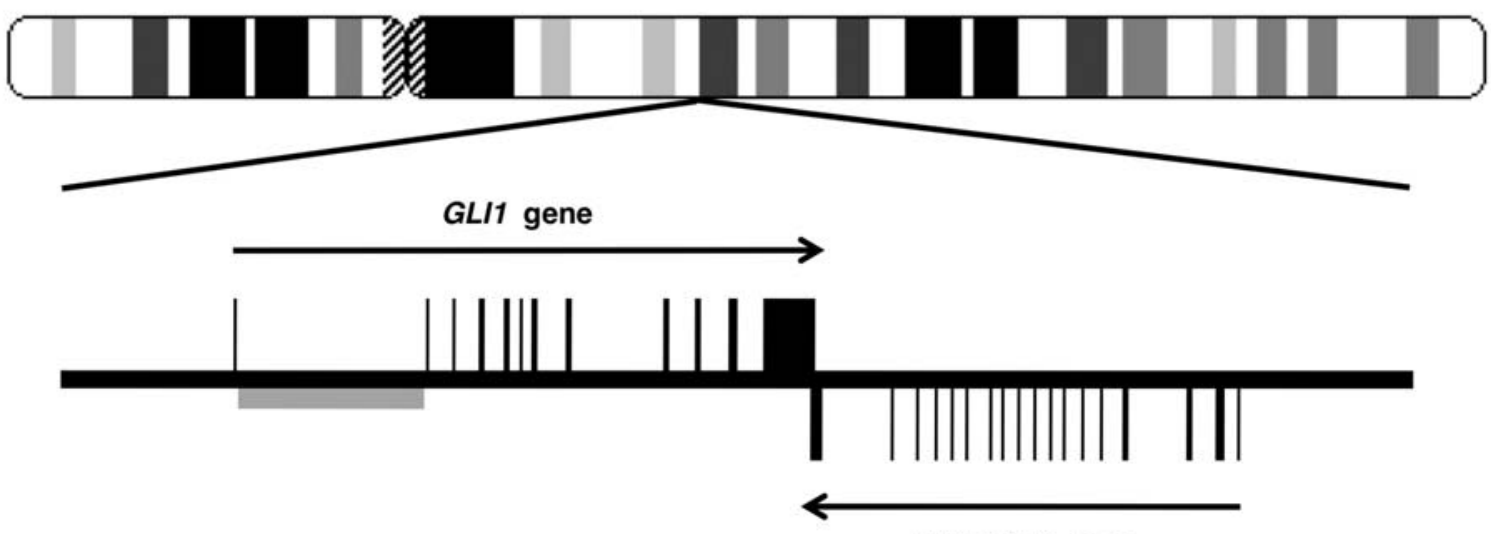

ARHGAP9 gene

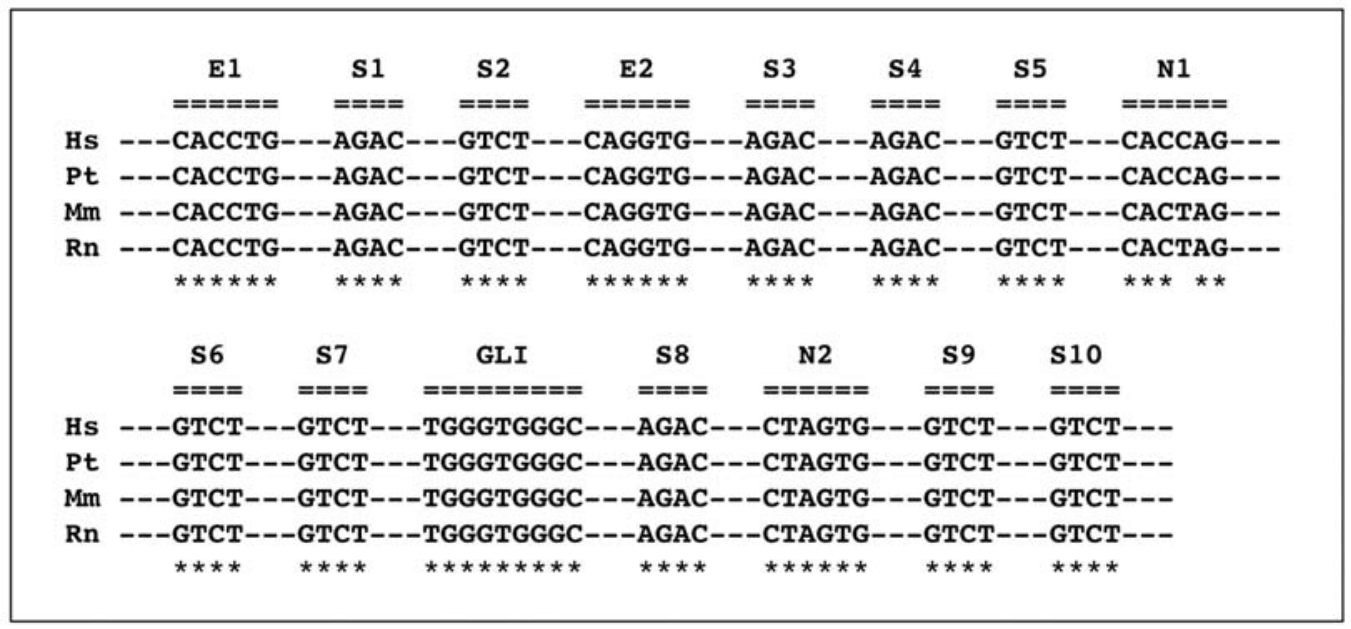

Figure 1. Integrative genomic analyses of GLI1. Schematic representation of GLII and ARHGAP9 genes at human chromosome 12q13.3 is shown in the upper part. GLII gene consisting of 12 exons and ARHGAP9 gene consisting of 18 exons are located in a tail-to-tail manner with overlapping $3^{\prime}$-ends. Conserved transcription factor-binding sites within intron 1 of GLI1 gene are shown in the gray box. E-boxes (E1 and E2), N-boxes (N1 and N2), Smad-binding elements (S1-S10), and GLI-binding site (GLI) are shown. Hs, human; Pt, chimpanzee; Mm, mouse; Rn, rat.

each other to give rise to double-stranded RNAs functioning as endogenous RNAi, GLII expression might be negatively regulated by the $A R H G A P 9$ transcripts.

Comparative genomic analyses of GLII orthologs. BLAST programs using human GLII genome sequence as a query sequence revealed that chimpanzee GLII gene, mouse Glil gene, and rat Glil gene were located within NW_001223153.1, AC114678.20, and AC122965.5 genome sequences, respectively. GLII orthologs were well conserved not only within exonic regions, but also within the proximal promoter region and intron 1 (data not shown).

Consensus GLI-binding element within the regulatory regions of human GLIl gene was searched for based on manual inspection, and a GLI-binding element with one base substitution was identified at the +1589 -bp position from the TSS (Fig. 1). The GLI-binding element was completely conserved in intron 1 of chimpanzee GLII, mouse Glil, and rat Glil genes (Fig. 1). Vokes et al carried out chromatinimmunoprecipitation assay combined with microarray analyses (ChIP-chip assay) of GLI-binding sites in mouse embryonic stem (ES) cells, and showed that the major peak of GLIbinding site within the regulatory regions of mouse Glil gene was located within intron 1 (41). We confirmed that the conserved GLI-binding element identified in this study was located within the genome fragment immunoprecipitated by using the anti-Gli1 antibody (data not shown). Therefore, it was concluded that the GLI-binding site at about $+1.5-\mathrm{kb}$ position from the TSS was evolutionarily conserved among mammalian GLII orthologs (Fig. 1).

Smad-binding element (SBE) within the regulatory regions of human GLI1 gene was next searched for based on manual inspection, and 10 SBEs within intron 1 of human GLII gene were found to be conserved in mouse Glil gene (Fig. 1). These 10 SBEs were also conserved in chimpanzee GLII and rat Gli1 genes (Fig. 1). TGFß signals induce Smad3-dependent upregulation of GLII and GLI2 in human NHDF fibroblasts, $\mathrm{HaCaT}$ keratinocytes, and MDA-MB-231 breast cancer cells (50); however precise mechanisms of Smad3-mediated GLI1 upregulation remained unclear. Conserved SBEs within intron 1 of mammalian GLII ortholgs clearly elucidated the mechanism of TGFß-induced GLII upregulation (Fig. 2).

Six bHLH-binding sites within intron 1 of human GLII gene were conserved in chimpanzee GLI1, mouse Glil, and rat Glil genes (data not shown). Two bHLH-binding sites located within the 5 '-region of intron 1 (Fig. 1) were the specific 


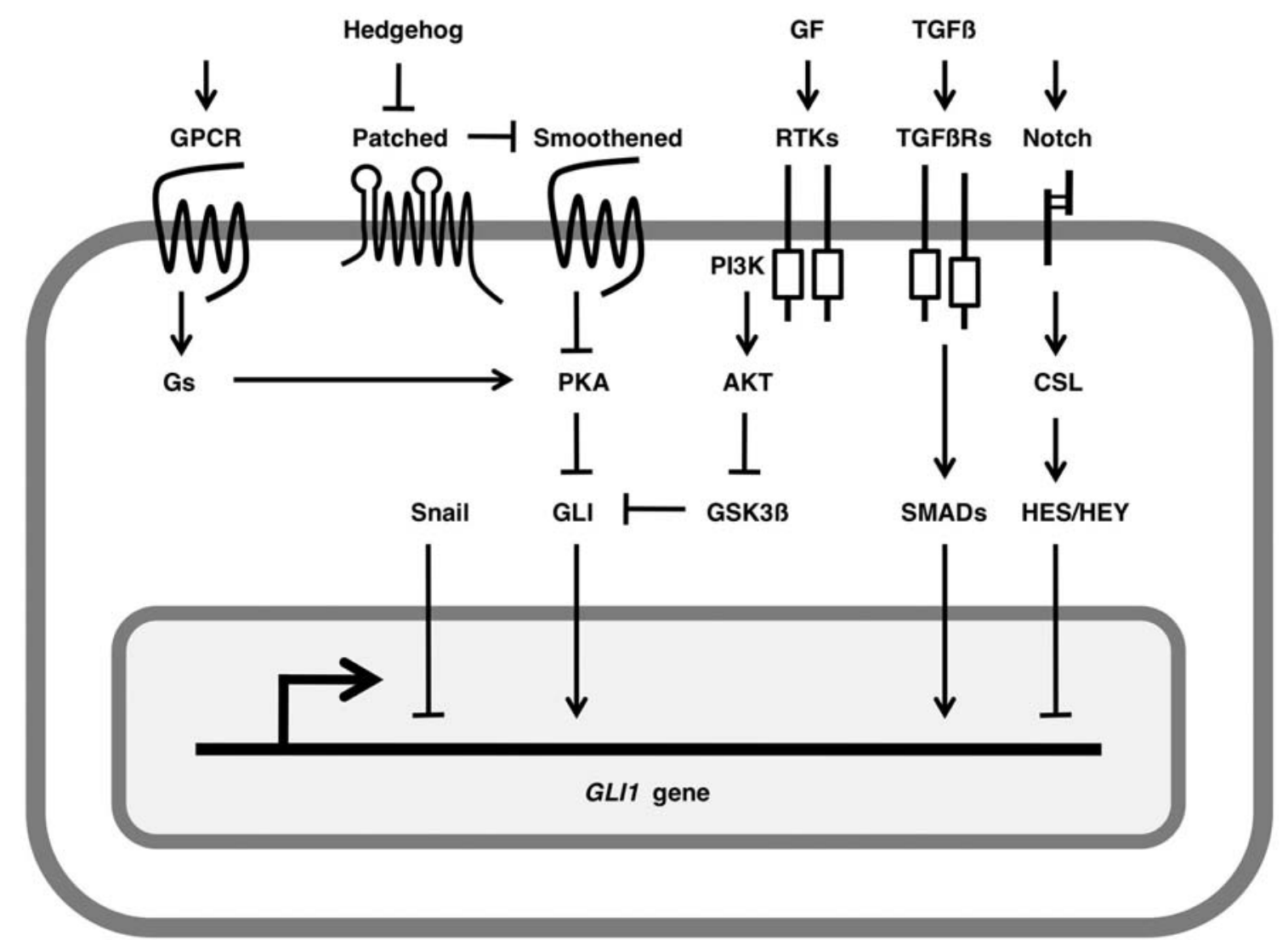

Figure 2. Stem-cell signaling network and GLI1 regulation. GLI1 is activated due to transcriptional activation as well as protein stabilization. Hedgehog and TGFß signals induce transcriptional upregulation of GLII, whereas Notch signals induce transcriptional downregulation of GLII. Receptor tyrosine kinase (RTK) signals via PI3K-AKT signaling cascade induce stabilization of GLI1 protein, whereas G-protein coupled receptor (GPCR) signals via Gs-PKA signaling cascade induce degradation of GLI1 protein.

E-boxes utilized as binding sites for TWIST1 (42). SNAI1/ Snail, SNAI2/Slug, ZEB1/§EF1/ZFHX1A, ZEB2/SIP1/ ZFHX1B, and TWIST2/DERMO1 also bind to the same E-boxes as TWIST1 to regulate the transcription of target genes (51-54). ZEB1, ZEB2, TWIST1, and TWIST2 function as transcriptional activators or repressors in a contextdependent manner, whereas SNAI1 and SNAI2 function as transcriptional repressors. Based on these facts, it was predicted that SNAI1 and SNAI2 repress GLII expression via the E-boxes (Fig. 2).

Double N-boxes within intron 1 of human GLII gene were also conserved in chimpanzee GLI1, mouse Gli1, and rat Glil genes (Fig. 1). Notch ligands induce Notch signaling activation through the NICD-CSL complex to upregulate Notch target genes, such as HES1, HES5, HEY1, HEY2 and HEYL (55-58). Consensus CSL-binding site was not conserved in mammalian GLII orthologs, whereas double N-boxes utilized as HES/HEY-binging sites within intron 1 of human GLI1 gene were conserved in chimpanzee GLI1, mouse Gli1, and rat Glil genes (Fig. 1). Together these facts indicate that Notch signals downregulate GLII expression via HES/HEY family members (Fig. 2).

\section{Discussion}

Integrative genomic analyses of GLII gene were carried out in this study. The GLII and ARHGAP9 genes are located in a tail-to-tail manner with overlapping 3'-ends (Fig. 1).
ARHGAP9 was expressed in bone marrow, spleen, thymus, monocytes, and macrophages, whereas GLII was almost undetectable in normal hematopoietic tissues or cells with predominant ARHGAP9 expression. Because overlapping sense and anti-sense transcripts are annealed to each other to give rise to double-stranded RNAs functioning as endogenous RNAi, GLII expression might be negatively regulated by ARHGAP9 transcripts.

GLI-binding element with one base substitution at the +1589-bp position from the TSS of human GLII gene was completely conserved in chimpanzee GLI1, mouse Gli1, and rat Glil genes (Fig. 1). Ten Smad-binding elements, double E-boxes and double N-boxes within intron 1 of human GLII gene were also conserved in mammalian GLII orthologs (Fig. 1). Hedgehog and TGFß signals induce transcriptional upregulation of target genes via GLI and Smads, respectively (Fig. 2). On the other hands, Snail/Slug and Notch-HES/HEY signals induce transcriptional downregulation of target genes via E-box and N-box, respectively (Fig. 2). GLII transcription is upregulated due to Hedgehog, and TGFß signaling activation, whereas GLI1 transcription is downregulated due to Snail/ Slug, and Notch signaling activation.

GLI1 is activated based on transcriptional activation as well as protein stabilization (Fig. 2). Hedgehog and TGFß signals induce transcriptional upregulation of GLI1, whereas Notch signals induce transcriptional downregulation of GLII. RTK signals via the PI3K-AKT signaling cascade induce stabilization of GLI1 protein, whereas G-protein coupled 
receptor (GPCR) signals via the Gs-PKA signaling cascade induce degradation of the GLI1 protein. Together these facts indicate that Hedgehog, TGFß, and RTK signals positively regulate GLI1, and that Notch, and GsPCR signals negatively regulate the GLI1 (Fig. 2).

Hedgehog, TGFß/BMP, RTK, Notch, and WNT signaling cascades constitute the stem-cell signaling network to orchestrate embryogenesis and carcinogenesis (59-63). Dysregulation of the stem-cell signaling network leads to GLI1 upregulation to promote carcinogenesis (Fig. 2). KAADcyclopamine and HhAntag are small-molecule compounds indirectly targeted to GLI1 and GLI2 via Smoothened $(64,65)$, whereas GANT61 is a small-molecule compound directly targeted to GLI1 and GLI2 (66). GLI-targeted small-molecule compounds could show anti-tumor effects to a broader range of cancer than Smoothened-targeted small-molecule compounds. To realize personalized medicine in the sequencing era with a peta-scale supercomputer (67), development of GANT61like compounds for clinical application is expected.

\section{References}

1. Marigo V, Roberts DJ, Lee SM, et al: Cloning, expression, and chromosomal location of $\mathrm{SHH}$ and $\mathrm{IHH}$. Genomics 28: 44-51, 1995.

2. Van den Brink GR, Bleuming SA, Hardwick JC, et al: Indian Hedgehog is an antagonist of Wnt signaling in colonic epithelial cell differentiation. Nat Genet 36: 277-282, 2004.

3. Garciadiego-Cazares D, Rosales C, Katoh M and ChimalMonroy J: Coordination of chondrocyte differentiation and joint formation by $\alpha 5 \beta 1$ integrin in the developing appendicular skeleton. Development 131: 4735-4742, 2004.

4. Katoh Y and Katoh M: Hedgehog signaling in gastric cancer. Cancer Biol Ther 4: 1050-1054, 2005.

5. Lum L and Beachy PA: The Hedgehog response network: sensors, switches, and routers. Science 304: 1755-1759, 2004.

6. Hooper JF and Scott MP: Communicating with Hedgehogs. Nat Rev Mol Cell Biol 6: 306-317, 2005.

7. Bailey J, Singh PK and Hollingsworth MA: Cancer metastasis facilitated by developmental pathways: Sonic hedgehog, Notch, and bone morphogenetic proteins. J Cell Biochem 102: 829-839, 2007

8. Katoh Y and Katoh M: Hedgehog signaling, epithelial-tomesenchymal transition, and miRNA. Int J Mol Med 22: 271-275, 2008

9. Kinzler KW, Bigner SH, Bigner DD, et al: Identification of an amplified, highly expressed gene in a human glioma. Science 236: 70-73, 1987.

10. Ruppert JM, Kinzler KW, Wong AJ, et al: The GLI-Kruppel family of human genes. Mol Cell Biol 8: 3104-3113, 1988

11. Bhatia N, Thiyagarajan S, Elcheva I, et al: Gli2 is targeted for ubiquitination and degradation by $\mathrm{B}-\mathrm{TrCP}$ ubiquitin ligase. J Biol Chem 281: 19320-19326, 2006

12. Tempé D, Casas M, Karaz S, et al: Multisite protein kinase A and glycogen synthase kinase $3 \beta$ phosphorylation leads to Gli3 ubiquitination by SCF BTrCP. Mol Cell Biol 26: 4316-4326, 2006.

13. Ruiz i Altaba A, Mas C and Stecca B: The Gli code: an information nexus regulating cell fate, stemness and cancer. Trends Cell Biol 17: 438-447, 2007.

14. Katoh Y and Katoh M: Integrative genomic analyses on GLI2: mechanism of Hedgehog priming through basal GLI2 expression, and interaction map of stem cell signaling network with P53. Int J Oncol 33: 881-886, 2008

15. Marigo V, Johnson RL, Vortkamp A and Tabin CJ: Sonic hedgehog differentially regulates expression of GLI and GLI3 during limb development. Dev Biol 180: 273-283, 1996.

16. Yoon JW, Kita Y, Frank DJ, et al: Gene expression profiling leads to identification of GLI1-binding elements in target genes and a role for multiple downstream pathways in GLI1-induced cell transformation. J Biol Chem 277: 5548-5555, 2002.

17. Hallikas O, Palin K, Sinjushina N, et al: Genome-wide prediction of mammalian enhancers based on analysis of transcription-factor binding affinity. Cell 124: 47-59, 2006.
18. Kasper M, Schnidar H, Neill GW, et al: Selective modulation of Hedgehog/GLI target gene expression by EGF signaling in human keratinocytes. Mol Cell Biol 26: 6283-6299, 2006.

19. Katoh Y and Katoh M: WNT antagonist, SFRP1, is Hedgehog signaling target. Int J Mol Med 17: 171-175, 2006.

20. He J, Sheng T, Stelter AA, et al: Suppressing Wnt signaling by the Hedgehog pathway through SFRP1. J Biol Chem 281: 35598-35602, 2006.

21. Yauch RL, Gould SE, Scales SJ, et al: A paracrine requirement of Hedgehog signaling in cancer. Nature 455: 406-410, 2008.

22. Roberts WM, Douglass EC, Peiper SC, Houghton PJ and Look AT: Amplification of the GLI gene in childhood sarcomas. Cancer Res 49: 5407-5413, 1989.

23. Werner CA, Döhner H, Joos S, et al: High-level DNA amplifications are common genetic aberrations in B-cell neoplasms. Am J Pathol 151: 335-342, 1997.

24. Kinzler KW, Ruppert JM, Bigner SH and Vogelstein B: The GLI gene is a member of the Kruppel family of zinc finger proteins. Nature 332: 371-374, 1988.

25. Dahmane N, Lee J, Robins P, Heller P and Ruiz i Altaba A: Activation of the transcription factor Gli1 and the Sonic hedgehog signalling pathway in skin tumours. Nature 389: 876-881, 1997.

26. Pomeroy SL, Tamayo P, Gaasenbeek M, et al: Prediction of central nervous system embryonal tumour outcome based on gene expression. Nature 415: 436-442, 2002.

27. Watkins DN, Berman DM, Burkholder SG, et al: Hedgehog signalling within airway epithelial progenitors and in small-cell lung cancer. Nature 422: 313-317, 2003.

28. Berman DM, Karhadkar SS, Maitra A, et al: Widespread requirement for Hedgehog ligand stimulation in growth of digestive tract tumors. Nature 425: 846-851, 2003.

29. Ma X, Chen K, Huang S, et al: Frequent activation of the Hedgehog pathway in advanced gastric adenocarcinomas. Carcinogenesis 26: 1698-1705, 2005.

30. Thayer SP, Di Magliano MP, Heiser PW, et al: Hedgehog is an early and late mediator of pancreatic cancer tumorigenesis. Nature 425: 851-856, 2003.

31. Ma X, Sheng T, Zhang Y, et al: Hedgehog signaling is activated in subsets of esophageal cancers. Int J Cancer 118: 139-148, 2006.

32. Kubo M, Nakamura M, Tasaki A, et al: Hedgehog signaling pathway is a new therapeutic target for patients with breast cancer. Cancer Res 64: 6071-6074, 2004.

33. Karhadkar SS, Bova GS, Abdallah N, et al: Hedgehog signalling in prostate regeneration, neoplasia and metastasis. Nature 431: 707-712, 2004

34. Patil MA, Zhang J, Ho C, Cheung ST, Fan ST and Chen X: Hedgehog signaling in human hepatocellular carcinoma. Cancer Biol Ther 5: 111-117, 2006.

35. Xuan YH, Jung HS, Choi YL, et al: Enhanced expression of Hedgehog signaling molecules in squamous cell carcinoma of uterine cervix and its precursor lesions. Mod Pathol 19: 1139-1147, 2006.

36. Feng YZ, Shiozawa T, Miyamoto T, et al: Overexpression of Hedgehog signaling molecules and its involvement in the proliferation of endometrial carcinoma cells. Clin Cancer Res 13: 1389-1398, 2007

37. Stecca B, Mas C, Clement V, et al: Melanomas require HedgehogGLI signaling regulated by interactions between GLI1 and the RAS-MEK/AKT pathways. Proc Natl Acad Sci USA 104: 5895-5900, 2007.

38. Liao X, Siu MK, Au CW, et al: Aberrant activation of Hedgehog signaling pathway in ovarian cancers: effect on prognosis, cell invasion and differentiation. Carcinogenesis 30: 131-140, 2009.

39. Zwerner JP, Joo J, Warner KL, et al: The EWS/FLI1 oncogenic transcription factor deregulates GLI1. Oncogene 27: 3282-3291, 2008.

40. Liu CZ, Yang JT, Yoon JW, et al: Characterization of the promoter region and genomic organization of $G L I$, a member of the Sonic hedgehog-Patched signaling pathway. Gene 209: 1-11, 1998.

41. Vokes SA, Ji H, McCuine S, et al: Genomic characterization of Gli-activator targets in Sonic hedgehog-mediated neural patterning. Development 134: 1977-1989, 2007.

42. Villavicencio EH, Yoon JW, Frank DJ, Fuchtbauer EM, Walterhouse DO and Iannaccone PM: Cooperative E-box regulation of human GLII by TWIST and USF. Genesis 32: 247-258, 2002

43. Katoh Y and Katoh M: Conserved POU-binding site linked to SP1-binding site within FZD5 promoter. Int J Oncol 30: 751-755, 2007. 
44. Katoh $\mathrm{M}$ and Katoh M: Comparative integromics on FZD7 orthologs. Int J Mol Med 19: 529-533, 2007.

45. Katoh M and Katoh M: Conserved POU/OCT- and GATAbinding sites in 5'-flanking promoter region of mammalian WNT8B orthologs. Int J Oncol 30: 1273-1277, 2007.

46. Katoh Y and Katoh M: Comparative integromics on JMJD2A, JMJD2B and JMJD2C. Int J Mol Med 20: 269-273, 2007.

47. Katoh $\mathrm{M}$ and Katoh $\mathrm{M}$ : Comparative integromics on noncanonical WNT or planar cell polarity signaling molecules. Int J Mol Med 20: 405-409, 2007.

48. Katoh Y and Katoh M: Transcriptional regulation of WNT2B based on the balance of Hedgehog, Notch, BMP and WNT signals. Int J Oncol 34: 1411-1415, 2009.

49. Furukawa Y, Kawasoe T, Daigo Y, et al: Isolation of a novel human gene, ARHGAP9, encoding a rho-GTPase activating protein. Biochem Biophys Res Commun 284: 643-649, 2001

50. Dennler S, André J, Alexaki I, et al: Induction of Sonic hedgehog mediators by TGF- 3 : Smad3-dependent activation of Gli2 and Gli1 expression in vitro and in vivo. Cancer Res 67: 6981-6986, 2007.

51. Rosivatz E, Becker I, Specht K, et al: Differential expression of the EMT regulators Snail, SIP1, and Twist in gastric cancer. Am J Pathol 161: 1881-1891, 2002.

52. Katoh M: Epithelial-mesenchymal transition in gastric cancer Int J Oncol 27: 1677-1683, 2005

53. Castro Alves C, Rosivatz E, Schott C, et al: Slug is overexpressed in gastric carcinomas and may act synergistically with SIP1 and Snail in the down-regulation of E-cadherin. J Pathol 211: 507-515, 2007.

54. Peinado H, Olmeda D and Cano A: Snail, Zeb and bHLH factors in tumour progression: an alliance against the epithelial phenotype? Nat Rev Cancer 7: 415-428, 2007

55. Artavanis-Tsakonas S, Rand MD and Lake RJ: Notch signaling: cell fate control and signal integration in development. Science 284: 770-776, 1999
56. Radtke $\mathrm{F}$ and Raj $\mathrm{K}$ : The role of Notch in tumorigenesis: oncogene or tumor suppressor? Nat Rev Cancer 3: 765-767, 2003.

57. Katoh $\mathrm{M}$ and Katoh $\mathrm{M}$ : Notch signaling in gastrointestinal tract. Int J Oncol 30: 247-251, 2007.

58. Katoh M and Katoh M: Integrative genomic analyses on HES/ HEY family. Int J Oncol 31: 461-466, 2007.

59. Katoh M: WNT and FGF gene clusters. Int J Oncol 21: 1269-1273, 2002.

60. Beachy PA, Karhadkar SS and Berman DM: Tissue repair and stem cell renewal in carcinogenesis. Nature 432: 324-331, 2004.

61. Radtke F, Clevers $\mathrm{H}$ and Riccio O: From gut homeostasis to cancer. Curr Mol Med 6: 275-289, 2006.

62. Katoh M: Dysregulation of stem cell signaling network due to germline mutation, SNP, Helicobacter pylori infection, epigenetic change, and genetic alteration in gastric cancer. Cancer Biol Ther 6: 832-839, 2007

63. Nusse R: Wnt signaling and stem cell control. Cell Res 18: 523-527, 2008.

64. Taipale J, Chen JK, Cooper MK, et al: Effects of oncogenic mutations in Smoothened and Patched can be reversed by cyclopamine. Nature 406: 1005-1009, 2000.

65. Romer JT, Kimura H, Magdaleno S, et al: Suppression of the Shh pathway using a small molecule inhibitor eliminates medulloblastoma in Ptc1(+/-)p53(-/-) mice. Cancer Cell 6: 229240, 2004.

66. Lauth M, Bergström A, Shimokawa T and Toftgård R: Inhibition of GLI-mediated transcription and tumor cell growth by smallmolecule antagonists. Proc Natl Acad Sci USA 104: 8455-8460, 2007.

67. Katoh M: Demand for user-friendly 'omics' web service utilizing next-generation supercomputer in the sequencing era. Mol Cancer Genet 1: 1-2, 2008 